\title{
Models for the oxidation of silicon
}

\author{
E. A. Lewis and E. A. Irene \\ Department of Chemistry 045A, University of North Carolina, Chapel Hill. North Carolina 27518
}

(Received 26 August 1985; accepted 17 September 1985)

\begin{abstract}
Since the 1960's many adaptations to the linear parabolic model for silicon oxidation have been proposed. For the purposes of process engineering, curve fitting procedures which are sometimes devoid of physical content but numerically precise are employed. However, a truly unified physical model which is in quantitative accord with all known facts is still lacking. In this review we will discuss the "facts" and newer models.
\end{abstract}

\section{INTRODUCTION}

The great technological importance of the process by which the surface of a single crystal wafer of $S i$ is converted to a thin film of amorphous $\mathrm{SiO}_{2}$ is derived from the resultant electrical stability of the oxidized Si surface. More than 25 years ago, it was demonstrated ${ }^{\prime}$ that the band gap states on a $\mathrm{Si}$ surface, which arise as a result of the unsatisfied bonds, could be reduced by about five orders of magnitude by the growth of adventitious $\mathrm{SiO}_{2}$ on the Si surface. Such a dramatic reduction in the electronically active surface states via oxidation renders the Si surface usable for high performance device applications. This important finding has led to the emergence of the field of planar integrated circuits and indeed microelectronics with $\mathrm{Si}$ as the preeminent semiconductor. Thus, since the early 1960 's there has been a considerable research and development effort, in order to better understand and improve the Si oxidation process.

As a result of the research activity, many Si oxidation models have been proposed. Some of the models have been based on a new fact or facts that have been uncovered in research. Other models have been derived through analogy with similar materials that have been carefully studied, and still other models have been proposed from curve fitting exercises where the concordant shapes of the model and the oxidation data in terms of $\mathrm{SiO}_{2}$ film thickness $L$ versus oxidation time $t$ are taken as confirmatory of the model. Most models are combinations of these methods. It is the purpose of this paper to review the Si oxidation modeling effort. Section II of this review will treat the commonly known facts about Si oxidation, such as, where the oxidation reaction takes place and characteristics of the oxide itself. Following this there will be a brief review in Sec. II of the early models which includes a description of the shape of the data and a discussion of the typical experimental apparatus. It will be shown that many aspects of the early models are still valid. However, new facts are constantly being added to the literature and these facts undoubtedly lead to revised and new models. Section IV will review some of the more recent studies which have added new facts for modeling and Sec. $V$ will cover new and/or revised models. The theme to be developed, is that while there has been considerable progress both in understanding the mechanism of Si oxidation and in developing the process technology, there still exists many important unanswered questions. At the present time the known facts are insufficient to unambiguously determine what is the actual Si oxidation mechanism. However, in many cases the presently available information can be used to eliminate some models and this will be discussed where appropriate. The final section will summarize the present status of Si oxidation modeling and suggest possible future directions.

\section{THE NATURE OF THE OXIDATION PROCESS}

Successful modeling of any reaction process requires a complete knowledge of the physical and chemical mechanisms by which the reaction proceeds. For the specific case of silicon oxidation, an insulating film of $\mathrm{SiO}_{2}$ is formed on a Si surface in the presence of an oxidizing ambient. Since the oxide formed separates the two reacting species, diffusion of one or both of the reactants through the oxide layer must occur prior to the chemical reaction to form $\mathrm{SiO}_{2}$. Therefore, modeling the Si oxidation process requires understanding the structural properties of the oxide in addition to the transport and chemical properties of the reactants since the oxide provides the medium through which the reaction process proceeds. Figure 1 illustrates the Si oxidation process, showing the diffusion and reaction sequence. It is well established that the oxidant is the diffusing species and that the reaction to form $\mathrm{SiO}_{2}$ occurs at the $\mathrm{Si}-\mathrm{SiO}_{2}$ interface. ${ }^{2-10}$ However, the details of the diffusion process and the nature of the oxide formed remain as valid research issues. Our current understanding of these two aspects of the oxidation process will be discussed below.

Characterizing the diffusion process involves not only identifying which reactant is diffusing, but also determining how the diffusing species interacts with the $\mathrm{SiO}_{2}$ network and what type of charge, if any, the oxidizing species possesses. Early studies indicated that oxygen was the diffusing species in dry oxidation processes ${ }^{3,5}$ while $\mathrm{H}_{2} \mathrm{O}$ was identified as the diffusing species for stearn ambients. ${ }^{4}$ Recent studies confirm and expand upon these early conclusions.

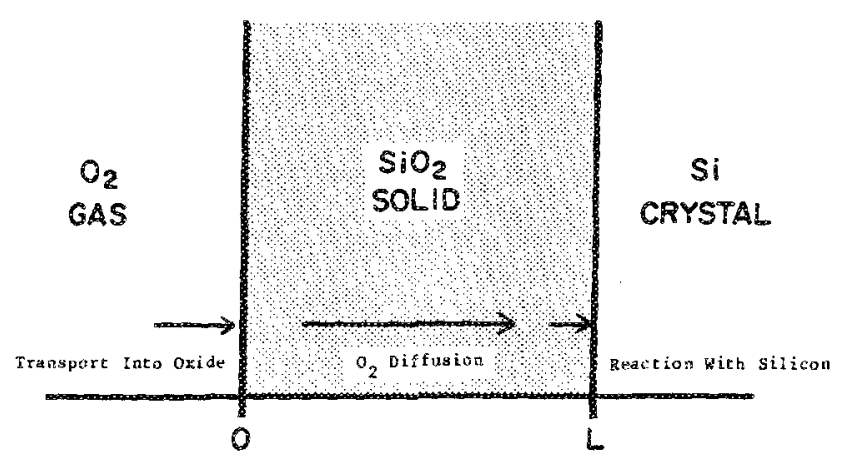

Fig. 1. The transport and reaction sequence for Si oxidation. 
Results from using ${ }^{18} \mathrm{O}_{2}$ to trace the oxidation process demonstrated that the oxidation proceeds predominantly by the long range migration of molecular oxygen without exchange of atomic $\mathrm{O}$ with the oxide network. ${ }^{6,9,10}$ The experiments were conducted by oxidizing $S i$ in an ${ }^{16} \mathrm{O}_{2}$ ambient, changing the ambient to ${ }^{18} \mathrm{O}_{2}$ and determining which oxide regions contained isotopic oxygen. An interesting result was that although about $93 \%$ of the isotopic $\mathrm{SiO}_{2}$ was located at the $\mathrm{Si}_{-} \mathrm{SiO}_{2}$ interface, indicating transport of $\mathrm{O}_{2}$ without exchange in the oxide, there was a thin layer of isotopic $\mathrm{SiO}_{2}$ at the outer surface. This outer layer containing ${ }^{18} \mathrm{O}$ is thought to be related to the motion of network oxygen atoms exchanging near the external surface. ${ }^{9,11}$ Although the effect of this mechanism on the overall transport process is small, its relative importance appears to increase in the very early stages of oxidation. ${ }^{9}$ Similar studies for oxidation in steam ambients ${ }^{7,8}$ concluded that transport in the $\mathrm{SiO}_{2}$ film is by interstitial, dissolved, molecular $\mathrm{H}_{2} \mathrm{O}$ but that a reversible exchange occurs between molecularly dissolved $\mathrm{H}_{2} \mathrm{O}$ and $\mathrm{Si}-\mathrm{O}$ bonds in the network. The charge state of the diffusing oxidant species has been a source of controversy for many years. Neutral $\mathrm{O}_{2}, \mathrm{O}_{2}^{-}$, and $\mathrm{O}_{2}^{-}$have been suggested as possible oxidant species for dry oxygen ambients. Reaction via a charged species versus a neutral species would significantly affect the oxidation kinetics particularly in the early stages of oxidation. ${ }^{2}$ Evidence for oxidation via a charged species was reported by Jorgenson ${ }^{4}$ in experiments which concluded that the oxidation process could be accelerated or retarded by the application of an electric field. However, Raleigh ${ }^{13}$ disputed this argument claiming that the same results would be obtained if the electric field had superimposed an electrolytic reaction upon the nomal oxidation reaction. Recent results from an experimental study of the effects of electric fields on the oxidation process by Modlin and Tiller, demonstrate that Jorgensen's results are not valid. ${ }^{14}$ Interfering reactions from the platinum electrodes used in Jorgensen's study negate the conclusions derived from that investigation. The Modlin and Tiller results, obtained by using a corona discharge to create the electric field and thereby eliminating metal contact with the oxide, concluded that the diffusing species is neutral for oxide thicknesses above 300 $\AA$. The transport mechanism for oxide growth up to $300 \AA$ has not been clearly determined. Thus, the best information to date is that for dry oxygen ambients the diffusing species is neutral $\mathrm{O}_{2}$ which does not interact with the oxide, while for wet ambients the diffusing species, molecular $\mathrm{H}_{2} \mathrm{O}$, rapidly exchanges with the $\mathrm{SiO}_{2}$ network.

The physical and structural properties of the oxide influence transport of the oxidizing species and the chemical reaction at the interface. Thus, the oxide structure becomes an important factor in governing the overall reaction kinetics. Understanding the effects of oxide structure on the oxidation process is essential to evaluating the kinetic behavior. Thermal oxidation produces a uniform, continuous film of $\mathrm{SiO}_{2}{ }^{1,15}$ on the Si surface. Electron diffraction results indicate that the $\mathrm{SiO}_{2}$ film possesses an amorphous network structure, ${ }^{1}$ although crystallites, identified as $\alpha$-crystobalite, have been observed. ${ }^{1,16}$ Recent investigations related to the oxide structure have centered on several key issues: the oxide structure at the $\mathrm{Si}-\mathrm{SiO}_{2}$ interface, the presence of micropores in the oxide, and the effect of stress, introduced at low temperatures, on the oxide structure.

The bulk oxide consists of a three-dimensional random network of $\mathrm{SiO}_{4}$ tetrahedra, ${ }^{17}$ but the structural properties of the oxide located within $30-40 \AA$ of the $\mathrm{Si}-\mathrm{SiO}_{2}$ interface are different and have been the subject of numerous investigations. ${ }^{18-25}$ It is well known and not surprising that the transition from crystalline $\mathrm{Si}$ to $\mathrm{SiO}_{2}$ is not sharp. Early studies ${ }^{18,19}$ indicated that a nonstoichiometric Si-rich oxide existed at the interface but could not accurately determine the extent and morphology of this region. Assimilating data from numerous TEM and spectroscopic experiments, the current understanding of the interface region is as follows. ${ }^{26}$ The transition from crystalline $S i$ to an amorphous oxide is rather abrupt (about $3 \AA$ ), but there is a transition region of Si-rich $\mathrm{SiO}_{2}$ which extends several monolayers into the amorphous oxide before a stoichiometric $\mathrm{SiO}_{2}$ structure is established. Exact measurements of the extent of this region are limited by interfering problems associated with the measurement techniques. Although the oxide attains a stoichiometric $\mathrm{SiO}_{2}$ composition within $5-10 \AA$ of the interface, there is an additional transition region, extending to approximately $30 \AA$, which does not possess the properties of bulk $\mathrm{SiO}_{2}$. This region appears to be strained, with a distribution of tetrahedral rings different from the bulk. Correlation of the structure and extent of the transition region with oxidation variables such as temperature and pressure has not been reported. Determining such a correlation may provide insight into factors affecting the oxidation reaction at the interface.

High resolution TEM studies have provided evidence for the existence of voids or "pores" in dry ${ }^{27,28}$ and wet ${ }^{28}$ grown $\mathrm{SiO}_{2}$ films. These voids, ranging from $5-10 \AA$ in diameter with a density of $10^{12} \mathrm{~cm}^{-2}$, are possibly elongated in the oxide. Although no direct evidence exists to indicate micropores extending the entire thickness of the fim, if they extend a significant distance into the oxide they could provide an aiternate mode of transport to the interface. ${ }^{29-31}$ There are also reports of microchannels in the oxicie which arise from local ordering of the $\mathrm{SiO}_{4}$ tetrahedra to form structural channels in the amorphous network. ${ }^{11}$ These microchannels are not related to the micropore structures discussed above which are gross structural defects involving broken bonds. No direct experimental evidence exists for microchannels nor has an oxidation model been proposed which utilizes these features.

The effect of stress on the oxide structure has become an issue of primary interest as the trend toward the use of lower oxidation temperatures increases. There is evidence that the bulk properties of the oxide grown at temperatures below $800^{\circ} \mathrm{C}$ are different from those of the high temperature oxides. ${ }^{64}$ The role of stress on the oxidation process will be discussed in detail in a later section, but it is important to emphasize that the physical properties of the oxide affect both the transport and reaction processes. Any changes in the structural properties of the oxide due to oxidation temperature or pressure will be reflected in the oxidation kinetics. 


\section{EARLY MODELS OF THE OXIDATION PROCESS}

As stated previously and shown in Fig. 1, the oxidation reaction proceeds by the transport of oxidant through the oxide followed by reaction with silicon at the $\mathrm{Si}-\mathrm{SiO}_{2}$ interface. The overall rate of the reaction will be governed by the slowest process. Very eariy attempts to model silicon oxidation produced much debate over which mathematical expression best represented the data. Numerous studies over a wide range of oxidizing temperatures and pressures used expressions of the form $L=k t^{0.5},{ }^{1,2,15,32-35} L=k \log$ $\times(1+a t),{ }^{33}$ or $L^{n}=k t,{ }^{36}$ to represent oxide growth data collected as thickness $L$ versus time $t$. These studies lacked a common basis for comparison and thus controversy over the description of the oxidation kinetics persisted. However, most studies in the temperature range $1000-1300^{\circ} \mathrm{C}$ agreed that the data could be reasonably well represented by the parabolic expression $L=k t^{0.5}$ which is consistent with a diffusion limited process. It was recognized, though, that the data adhered to a strictly parabolic expression only for oxidation temperatures above $1000^{\circ} \mathrm{C}$ and oxide thicknesses greater than $2000 \AA .{ }^{15}$ For lower temperatures and thin oxides a mixed linear-parabolic expression $k_{1} L^{2}+k_{2} L=t$ was required to represent the data. This mixed linear-parabolic form indicated that the oxidation was changing from a purely diffusion limited reaction to one that was also controlled by the interface reaction. In a 1965 paper, which has become a classic in the field, Deal and Grove ${ }^{37}$ presented a comprehensive study of oxidation kinetics over a broad range of temperatures $\left(700-1200^{\circ} \mathrm{C}\right)$ and pressures $(0.1-$ $1.0 \mathrm{~atm}$ ) in both wet and dry ambients. The model they proposed to explain the linear-parabolic nature of the data has served as the basis for the interpretation of all subsequently collected Si oxidation data. The model involves a steady state analysis of the physical and chemical processes known to occur during the reaction. Figure 2 shows the three processes considered in the model: (1) transport of the oxidizing species across the ambient-SiO $\mathrm{O}_{2}$ interface, (2) Fickian difiusion through the oxide, and (3) reaction with Si at the $\mathrm{Si}-\mathrm{SiO}_{2}$ interface. Under steady state conditions these three fluxes, occurring in series, will be equal at all times with

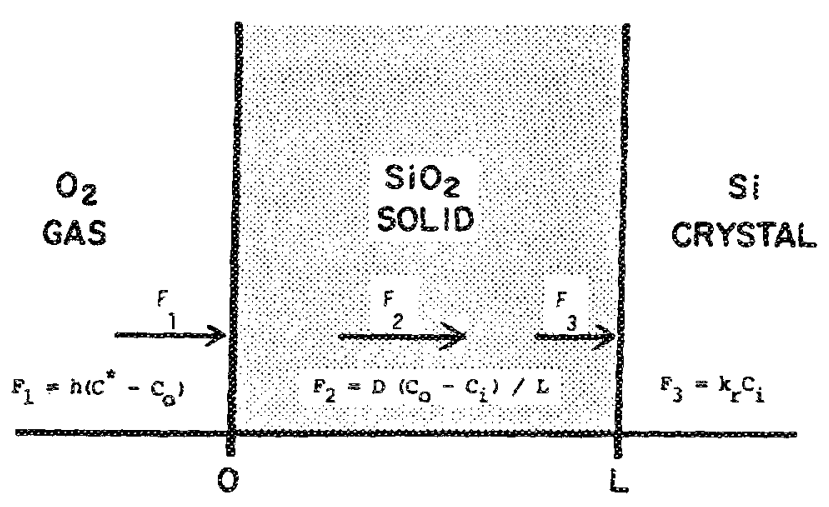

Fra. 2. The Deal-Grove model. $\mathrm{h}$ is the gas-phase transport coefficient, $\mathrm{C}^{*}$ is the equilibrium concentration of $\mathrm{O}_{2}$ in $\mathrm{SiO}_{2}, \mathrm{C}_{\mathrm{o}}$ is the concentration of $\mathrm{O}_{2}$ at the outer $\mathrm{SiO}_{2}$ surface, $\mathrm{C}_{\mathrm{i}}$ is the concentration of $\mathrm{O}_{2}$ at the $\mathrm{Si}-\mathrm{SiO}_{2}$ interface, and $\mathrm{D}$ is the effective diffusion constant for $\mathrm{O}_{2}$ in $\mathrm{SiO}_{2}$.

$$
F_{1}=F_{2}=F_{3} .
$$

The oxidation rate is given by the equation

$$
d L / d t=F / \Omega,
$$

where $\Omega$ represents the number of oxidant molecules incorporated into a unit volume of oxide. Using the flux equations presented in Fig. 2, the integrated form of the rate equation is

$$
\frac{\left(L^{2}-L_{0}^{2}\right)}{k_{p}}+\frac{\left(L-L_{0}\right)}{k_{1}}=t-t_{0}
$$

where $k_{p}=2 D C * / \Omega$ and $k_{1}=k_{r} C^{*} / \Omega$ represent physicochemical parameters of the reaction. Here, $D$ is the effective diffusion constant for transport through the oxide, $C^{*}$ is the equilibrium concentration of oxidant in the $\mathrm{SiO}_{2}$, and $k_{r}$ represents the reaction rate constant for the conversion of $\mathrm{Si}$ to $\mathrm{SiO}_{2}$. Transport across the ambient-SiO ${ }_{2}$ interface is much faster than the other two fluxes and thus the transport coefficient $h$ does not affect the reaction rate constants $k_{p}$ or $k_{1}$. The parabolic rate constant $k_{p}$ is related to diffusion through the oxide while the linear rate constant $k_{1}$ directly reflects the reaction at the Si surface. The constants $L_{0}$ and $t_{0}$ are offsets to the Deal-Grove model which account for an initial region in dry $\mathrm{O}_{2}$ oxidations, extending to about $300 \AA$, where the model does not accurately describe the experimental data. An offset is not required when wet ambients are used. Aside from the initial regime in dry $\mathrm{O}_{2}$ ambients, the model showed excellent agreement for both steam and dry oxidation data. The oxidation rate in the initial regime is more rapid than predicted by the Deal-Grove model and has been the subject of numerous investigations over the past 20 years.

Thus, although the Deal-Grove model was a significant contribution to the interpretation of Si oxidation data, it is not complete and needs revisions particularly for the initial stages of dry oxidation kinetics. Other problems associated with the model will be discussed in the next section. The importance of the initial regime increases as the oxidation temperature is lowered, since at temperatures below $800^{\circ} \mathrm{C}$ the oxide grown in a reasonable time is entirely within the thickness of the initial regime. The current trend towards the use of thinner films and lower processing temperatures has intensified the drive to explain the oxidation kinetics in this thickness regime. ${ }^{38}$

Before proceeding with the next section it may be useful to briefty describe the procedure used for studying the thermal oxidation of silicon. A typical oxidation is performed in a double walled quartz furnace tube, as shown in Fig. 3, at temperatures ranging from $800-1200^{\circ} \mathrm{C}$. The furnace temperature is set, equilibrated and calibrated prior to introducing the Si wafers. By flowing an inert gas such as nitrogen through the outer jacket of the oxidation tube the diffusion of impurities such as $\mathrm{Na}^{+}$and $\mathrm{H}_{2} \mathrm{O}$ is minimized. An inert ambient, usually nitrogen or argon, flows through the inner tube prior the oxidation to minimize the growth of any oxide during the time it takes for the samples to reach the temperaure of the ambient. The cleaning procedure for the wafers 


\section{Silicon Oxidation System}

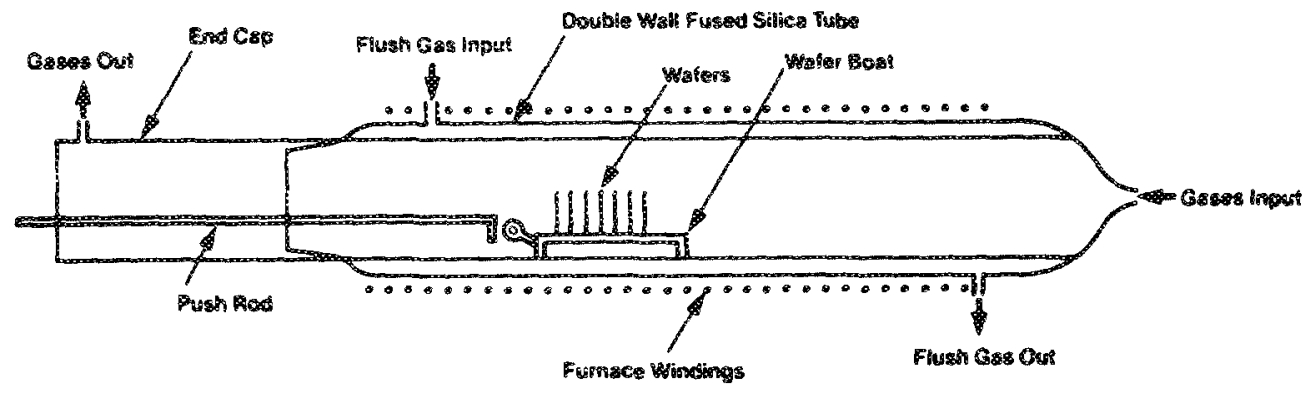

FIG. 3. Thermal oxidation apparatus (from Ref. 91, Fig. 5, with permission).

before entry into the oxidation furnace is rigorous in order to preclude the effects of impurities on the oxidation process. The samples are then placed on a quartz holder, entered into the inner tube of the furnace, and pushed to the center of the hot zone. After allowing the samples to attain the desired oxidation temperature (approximately $15 \mathrm{~min}$ ), the oxidizing ambient is introduced into the inner tube. For dry oxida. tions this will be pure, dry oxygen while for wet oxidations the oxygen is first bubbled through deionized water. The oxidation is then allowed to proceed for the desired time interval. Measurement of the $\mathrm{SiO}_{2}$ film thickness is usually performed by ellipsometry, an optical technique based on changes in the state of polarization of an incident monochromatic light beam upon refiection from the film covered Si surface. The advent of the use of in situ ellipsometric studies, where an oxidation funace is mounted at the focus of an ellipsometer, has been an important development in studying Si oxidation kinetics. ${ }^{39-41}$ This in situ setup enables the collection of dense thickness versus time data sets and therefore allows an accurate determination of the rate constants.

\section{U. NEW FACTS RELATED TO THE OXIDATION PROCESS}

The Deal-Grove mode ${ }^{37}$ has repeatedly provided an excellent fit to Si oxidation data. ${ }^{40-49}$ However, in addition to the unexplained initial regime, detailed studies of various aspects of the oxidation process have uncovered new facts which are either inconsistent with the Deal-Grove model or not accourted for in the model. The use of in situ ellipsometric studies has been instrumental in revealing details of the oxidation mechanism. These studies and others support the Deal-Grove model as a basis for analyzing Si oxidation data, but indicate that the oxidation process is more complex than originally proposed. In this section the properties of the rate constants and overall reaction rate predicted from the model will be discussed and compared with new facts derived from observed experimental behavior. Attempts to incorporate the new facts into models of the oxidation process will be discussed in the next section.

In the Deal-Grove model the parabolic rate constant $k_{p}=2 D C * / \Omega$ reflects the diffusional transport of oxidant through the oxide. If $k_{p}$ corresponds to an elementary, thermally activated process obeying Boltzman statistics, its temperature dependence should be described by an Arrhenius equation of the form

$$
k_{p}=k_{0} \exp \left(-E_{a} / k_{B} T\right),
$$

where $k_{B}$ is the Boltzman constant, $T$ is the oxidation temperature, and $E_{a}$ is the single activation energy for the process which in this case is diffusion through the oxide. For a kinetically simple rate process described by a single rate constant, say $k_{p}$, the Arrhenius plot, $\ln \left(k_{p}\right)$ versus $1 / T$, will be a straight line with a slope equal to the activation energy. Deal and Grove showed that the $k_{p}$ values derived from their experimental results exhibited Arrhenitus behavior with activation energies determined for wet and dry oxidizing ambients that were similar to those measured for the diffusion of water and oxygen, respectively, in fused silica. They also reported a linear pressure dependence for $k_{p}$ over the range $0.1-1.0 \mathrm{~atm}$ from an analysis of literature data. This linear pressure dependence is concordant with the model since $k_{p}$ is directly proportional to $C *$, the equilibrium concentration of oxidant in the oxide. According to Henry's law, in the absence of dissociation, the equilibrium concentration, and thus $k_{p}$, will be linearly related to pressure. The pressure dependence reported for $k_{p}$ is therefore consistent with diffusion via a molecular species. Deal and Grove concluded that the mocel successfully predicted the temperature and pressure behavior of $k_{p}$. Further studies have confirmed the linear pressure dependence of $k_{p}$ at temperatures ranging from $870-1200^{\circ} \mathrm{C} .^{41,50}$ There is some question about the pressure dependence at lower temperatures, but the limited growth rate makes it difficult to determine rate constants accurately. The behavior of $k_{p}$ with temperature has been carefully studied and it is now apparent that $k_{p}$ does not exhibit the simple Arrhenius behavior originally reported in the Deal-Grove study. ${ }^{48,49,51}$ While the activation energies reported in the Deal-Grove study are valid for oxidation temperatures above $1000^{\circ} \mathrm{C}$, these new low temperature studies show a change in the slope of the Arrhenius plot occurring in the range $900-1000{ }^{\circ} \mathrm{C}{ }^{48,52}$ At lower temperatures the slope of the curve (i.e., the activation energy) increases and thus the shape of the plot is slightly concave downwards. This curvature is consistent with the shape of an Arrhenius plot for a complex rate constant describing two processes with different activation energies occurring in parallel. However, it does not prove that two transport mechanisms are involved. $k_{p}$ was observed to be orientation independent at temperatures above $1000^{\circ} \mathrm{C}^{42}$ as anticipated for diffusion through an amorphous oxide. However, at lower temperatures $k_{p}$ varies with Si orientation for both wet and 
dry oxidizing ambients. ${ }^{47,49}$ Thus it appears that the diffusion process or the structure of the oxide is influenced by the surface structure of the Si.

The linear rate constant $k_{1}=k_{r} C^{*} / \Omega$ should be a direct reffection of the reaction at the surface. Any changes in the reaction rate constant $k_{r}$ will directly affect $k_{1}$. Deal and Grove reported that $k_{1}$, in addition to $k_{p}$, exhibited Arrhenius behavior. The activation energy determined for $k_{1}$ was similar to the $\mathrm{Si}-\mathrm{Si}$ bond energy indicating that this bond breaking step was rate limiting. The Deal-Grove model also predicts a linear pressure dependence for $k_{1}$ since it is proportional to $C^{*}$. New findings present a more complex picture of the linear rate constant; the Arrhenius plot for $k_{1}$ is not linear ${ }^{45,48,49}$ and $k_{1}$ does not exhibit a linear pressure dependence. ${ }^{41,46,49}$ The Arrhenius plot for $k_{,}$, like the plot for $k_{p}$, exhibits a break in the slope occurring between 900 and $1000^{\circ} \mathrm{C}$, but the direction of curvature is opposite to that for $k_{p}$. A higher activation energy is observed for $k_{1}$ at temperatures above $1000^{\circ} \mathrm{C}$ than for lower temperatures. $45,48,49$ This shape, concave upward, parallels the shape of an Arrhenius plot for a complex rate constant representing processes with different activation energies occurring in series. ${ }^{52}$ Again, the shape of the curve is not sufficient evidence to prove this type of complex mechanism exists. The pressure dependence of $k_{1}, P^{n}$, varies with temperature from $n=0.59$ at low temperatures to $n=0.83$ at high temperatures. ${ }^{45}$ The exponent $n$ varies with orientation for a fixed temperature, which also indicates that $k_{1}$ does not represent a simple reaction step. ${ }^{45}$ This complex pressure dependence has been explained in terms of reaction by both molecular and atomic species at the interface ${ }^{54}$ Additionally, $k_{1}$ exhibits a distinct orientation dependence as a function of temperature ${ }^{45,47,49}$ at $1 \mathrm{~atm}$. $1 \mathrm{t}$ has been proposed ${ }^{53}$ that the relative ordering of $k_{1}$ values is related to the number of Si-Si bonds on the surface of a particular plane with $(110)>(111)>(100)$. However, this relative ordering of $k_{1}$ values only holds for temperatures above $900^{\circ} \mathrm{C}^{47,49}$ and thus other factors are also involved.

Interestingly, both $k_{p}$ and $k_{1}$ exhibit breaks in their Arrhenius plots in the temperature range $900-1000^{\circ} \mathrm{C}$, although the direction of curvature is opposite for the two cases. This change in siope reflects a change in the oxidation mechanism at low temperatures. As previously mentioned, it could indicate the presence of additional processes occurring in parallel or in series, respectively, with the diffusion and reaction fluxes stated in the Deal-Grove model. ${ }^{52}$ If additional fluxes are not present, then the curvature could reflect a thermally controlled property influencing the transport and reaction steps described in the model. Evidence for a temperature dependent stress affecting the kinetic behavior is available and will now be considered. Upon cooling from the oxidation temperature, a stress develops in the oxide due to differences in the thermal coefficients of expansion for $\mathrm{Si}$ and $\mathrm{SiO}_{2}{ }^{55}$ The temperature reported for relaxation of this thermal stress through viscous flow is around $9700^{\circ} \mathrm{C} .{ }^{55} \mathrm{This}$ temperature lies precisely within the range where a break occurs in the Arrhenius plots for both $k_{1}$ and $k_{p}$, and thus indicates viscous flow may play an important role in the oxidation process. Further studies revealed that in addition to the ther- mal stress an intrinsic stress, on the order of $10^{9} \mathrm{dyn} / \mathrm{cm}^{2}$, exists in oxides grown in both wet ${ }^{56}$ and dry ${ }^{56,57}$ ambients at temperatures below $950^{\circ} \mathrm{C}$. The intrinsic stress is a stress component which exists at the oxidizing temperature where the thermal stress component is zero. This intrinsic stress probably arises because the oxide lacks the ability for viscous flow to occur at these temperatures, ${ }^{57}$ and is not observed at oxidizing temperatures above $975^{\circ} \mathrm{C} 0^{56}$ Thus, a new parameter, intrinsic stress, is introduced into the oxidation process in the temperature range $950-975^{\circ} \mathrm{C}$ and is present at all lower oxidation temperatures. Although no direct correlation has been made between stress and the rate constants, the effects of stress on the oxidation reaction have been considered. ${ }^{31,58-61}$ It has also been reported that the refractive index of thermal $\mathrm{SiO}_{2}$ increases as the oxidation temperature is lowered. ${ }^{57,62,63}$ This indicates a structural change in the oxide as the temperature is lowered. Annealing the oxide in an inert ambient at temperatures where viscous flow can occur allows the oxide to relax to a refractive index characteristic of high temperature thermal oxides. ${ }^{57}$ The higher refractive index was originally attributed to an increased oxide density, ${ }^{57,62,63}$ but very recent results indicate the refractive index change may be caused by an incomplete oxidation leaving a Si-rich oxide. ${ }^{64}$ Thus, as discussed previously, the nature of the oxidation process depends on the properties of the oxide. The presence of stress and altered structural properties introduced at low temperatures could infuence transport and the reaction. Their effect on the kinetic mechanism must be determined in order to develop a comprehensive model for the oxidation process.

Other observations have been made about the overall reaction kinetics in terms of the Deal-Grove model. Determining the pressure dependence of $k_{p}$ and $k_{1}$ individually at low pressures is difficult since the limited oxide growth precludes extracting accurate rate constants. Therefore, some studies have investigated the pressure dependence of the overall reaction rate. ${ }^{41,46,49}$ According to the Deal-Grove model the oxidation rate should be linearly proportional to pressure over all thickness ranges. However, while the pressure dependence of $d L / d t$ is found to be linear for oxides greater than $300 \AA,{ }^{41}$ for oxides less than $300 \AA$ various studies have reported a pressure dependence ranging from $P^{0.5}$ to $P^{0.8}{ }^{41,46,49}$ Another interesting observation of Si oxidation involves the relative rates at which the various crystal orientations oxidize as a function of oxidation temperature, pressure, and time. For all temperatures in the range 800$1000^{\circ} \mathrm{C}$ the (100) Si orientation has the slowest oxidation rate $^{47,65}$ at $1 \mathrm{~atm}$. However, there is a crossover in the relative rate of oxidation between the (111) and (110) surfaces at each temperature in this range. ${ }^{65}$ In all cases the (110) orientation has the greatest initial oxidation rate, but at a thickness characteristic of the oxidation temperature the oxidation rate on the (111) surface becomes greater than on the (110) surface. ${ }^{65}$ The relative rates at very low pressures as a function of temperature is quite complex..$^{65,66}$ Further research on the relative oxidation rates involving additional orientations is presently underway in our laboratory.

Information on the effects of impurities on the oxidation process has been collected and may provide some insight 
into the oxidation mechanism. During the evolution of integrated circuit (IC) technology the emphasis on oxidation impurity effects has changed. The earliest studies were aimed at devising processing methods that would eliminate those impurities that degrade $1 \mathrm{C}$ device performance. The most notable impurity was sodium. $\mathrm{Na}^{+}$can diffuse quite rapidly in $\mathrm{SiO}_{2}$ and with its prodigious natural abundance is a major $\mathrm{Si}_{-}-\mathrm{SiO}_{2}$ interfacial impurity giving rise to large amounts of positive charge at the Si surface. In terms of oxidation kinetics, $\mathrm{Na}^{+}$has been found to enhance the Si oxidation rate. ${ }^{67}$ The details of the kinetics are not well known.

$\mathrm{H}_{2} \mathrm{O}$, as both an impurity and as an oxidant has received considerable attention. ${ }^{47,69}$ As an oxidant, $\mathrm{H}_{2} \mathrm{O}$ is a more virulent oxidant for $\mathrm{Si}$ than is $\mathrm{O}_{2}$. Hence, $\mathrm{H}_{2} \mathrm{O}$ is used for processing when thick oxides are required so as to reduce both the process time and temperature. However, the use of $\mathrm{H}_{2} \mathrm{O}$ gives rise to a large number of bulk oxide charge trapping centers which, when charged, also alter the Si surface potential as does $\mathrm{Na}^{+}$at the interface and affects the electric reliability of the device. ${ }^{68} \mathrm{H}_{2} \mathrm{O}$ oxidation kinetics have been studied in greater depth than for $\mathrm{Na}^{+}$. It was found that ever traces of $\mathrm{H}_{2} \mathrm{O}$ in $\mathrm{O}_{2}$ increase the rate of oxidation. ${ }^{47.69}$ Both $k_{1}$ and $k_{p}$ are enhanced. For the overall rate of oxidation the amount of enhancement was found to be greater than for $\mathrm{O}_{2}$ and $\mathrm{H}_{2} \mathrm{O}$ present as independent oxidants, i.e., there is a cooperative effect. ${ }^{69}$ Essentially, this arises from the fact that $\mathrm{H}_{2} \mathrm{O}$ reacts with the $\mathrm{SiO}_{2}$ network forming $\mathrm{SiOH}$ species and thereby terminating the Si-O-Si chains. This loosened network enables rapid diffusion of oxidant species whether the species are related to either $\mathrm{O}_{2}$ or $\mathrm{H}_{2} \mathrm{O}$. $\mathrm{H}_{2} \mathrm{O}$ has also been found to seriously modify the transport of $\mathrm{O}_{2}$ in other ways. Several complementary studies ${ }^{6-8,70} \mathrm{dem}-$ onstrate that pure $\mathrm{O}_{2}$ diffuses through the interstices in the $\mathrm{SiO}_{2}$ network. For the purpose of explaining Si oxidation there is no exchange of $\mathrm{O}$ with the $\mathrm{SiO}_{2}$ network. However, with even traces of $\mathrm{H}_{2} \mathrm{O}$ present $\mathrm{O}$ exchanges rapidly with the $\mathrm{SiO}_{2}$ network, hence dramatically altering the transport of oxidant and the oxidation mechanism.

Other impurities added to the oxidizing ambient have been considered such as $\mathrm{HCl}, \mathrm{Cl}_{2}$, and organic chlorine containing compounds. ${ }^{71-73}$ The literature attests to the fact that oxide charge levels are reduced and oxide reliability is enhanced through these additions. The effects on Si oxidation kinetics are about the same, i.e., all of these impurities increase the rate of oxidation. The explanation is likely the same in all of these cases. The impurities all react with the $\mathrm{SiO}_{2}$ network thus enabling more rapid transport of oxidant to the $\mathrm{Si}-\mathrm{SiO}_{2}$ interface where oxidation takes place.

Impurities or dopants added to the Si substrate also alter the oxidation kinetics. The most common Si dopants are $n$ type dopants such as $\mathrm{P}$ or As and p-type dopants such as B. Both kinds of dopants have been found to alter the Si oxida. tion kinetics but with different temperature dependencies. ${ }^{48,74}$ At oxidation temperatures of less than $1000^{\circ} \mathrm{C}$, the $n$-type dopants enhance the overall oxidation rate while at higher temperatures $B$ doping results in a higher rate. It appears that both $k_{1}$ and $k_{p}$ are affected. The $k_{p}$ effects are likely due to alterations of the $\mathrm{SiO}_{2}$ network as a result of bonding defects. The dopants $B, P$, and As are network formers but the resultant networks are different in structural arrangement and/or number of bonds to $0 .{ }^{48} \mathrm{~B}$ forms a planar trigonal arrangenent of $O$ 's while $P$ and As form four bonds to $O$ as does Si but the extra pair of nonbonding electrons distorts the tetrahedron. Such defects are iikely to enhance diffusion of the oxidant. The interfacial oxidation kinetics are even more complex. One model ${ }^{75}$ wtilizes the production of point defects at the interface to explain the enhanced rate for $\mathrm{P}$ doping. The Si band gap modifications were shown to increase the vacancy production and this scaled with the oxidation rate enhancement. Another new model for surface rate enhancement has been proposed. ${ }^{76} \mathrm{In}$ this model the availability of electronic carriers is the important property. For the case of submonolayer gold coverage on Si, it was shown that the unannealed surface was metallic while annealing ordered the surface and the ordering gave rise to a semiconductorlike surface. The metallic surface oxidized considerably more rapidly than the semiconductor surface presumably due to the availability of electrons for the formation of bonds. Interestingly, the oxidation of metal silicides also shows similar behavior. ${ }^{77}$ When a metal silicide film is grown directly on $\mathrm{Si}$, the silicide will oxidize to form $\mathrm{SiO}_{2}$ only with the preservation of the silicide film. In effect there is a net flow of Si to the silicide-oxidant interface. It was discovered that the metalliclike silicides oxidized much more rapidly than the semiconductorlike silicides and these latter silicides oxidized with similar rates as Si itself. It seems that several experimental studies have shown the importance of the electrical nature of the oxidizing surface. Such effects have largely been ignored in many oxidation modeis.

It is apparent that a considerable amount of information has been collected in the past 20 years giving more insight into the details of the oxidation process. These investigations have not only determined complex behavior in the rate constants but have also recognized the infuence of additional physical phenomena introduced at low temperatures on the oxidation reaction. Any mode! which provides a complete description of the oxidation process must be able to account for the observed experimental facts. It is clear that new models or revisions to the Deal-Grove rnodel are necessary to provide a comprehensive description of the oxidation mechanism.

\section{NEW MODELS FOR SI OXIDATION}

\section{A. Structural mode!}

Considering the recent emphasis on the effects of intrinsic stress on Si oxidation, Tiller ${ }^{78}$ proposed a model that minimizes the stress and hence the free energy required for the oxidation process. Essentially Tiller considers oxidation to be a two step process. In the first step $S i$ is converted to $\alpha$ cristobalite plus $\mathrm{Si}$ interstitials in both $\mathrm{Si}$ and $\mathrm{SiO}_{2}$. The $\alpha$. cristobalite lattice fits best on a Si lattice except for the presence of a few Si atoms, and thus yields the smallest molar volume change during oxidation. This first step minimizes the strain and hence the stress. In the second step the Si interstitials in the $\mathrm{SiO}_{2}$ are oxidized. The volume expansion inside the crystalline $\alpha$-cristobalite lattice causes distortion 
of the lattice and eventual amorphization. One implication of this model is that a layer of crystalline $\mathrm{SiO}_{2}$ should always be found at the $\mathrm{Si}-\mathrm{SiO}_{2}$ interface. While there is no overwhelming evidence for this, Aguis et al. ${ }^{79}$ have reported that under low oxidant pressures a crystalline modification of $\mathrm{SiO}_{2}$ is observed. Additionally, virtually all the modern surface spectroscopies(see for example, Refs. 18,19,26) agree that the interfacial oxide is a suboxide of $\mathrm{SiO}_{2}$. Some recent work in our laboratory ${ }^{64}$ on the structure of low temperature grown thermal oxide on Si shows that suboxides are formed in greater amounts when the stress is highest, i.e., at the lowest oxidation temperatures. We propose that the oxidation system accomodates to the accumulation of intrinsic stress which arises from the large molar volume change by rejecting complete oxidation and thereby reducing somewhat the large molar volume change. Mott ${ }^{80-82}$ has considered that if oxidation of $\mathrm{Si}$ proceeds at kink sites in the $\mathrm{Si}$ surface, then the large volume requirement is diminished and allows oxidation to proceed. The applicability of this idea has not been determined, but it is worthwhile to consider, since this mechanism may result in small strains that diminish with increasing temperature. This model may therefore provide an alternate explanation to the viscous fiow model which will be introduced in the section on stress models.

\section{B. Interface kinetics models}

Two interface kinetics models have received attention. ${ }^{54,83}$ The earlier Ghez and van der Meulen model ${ }^{54}$ is based on the reaction between $\mathrm{Si}$ and oxidant at the $\mathrm{Si}-\mathrm{SiO}_{2}$ interface involving both $\mathrm{O}_{2}$ and $\mathrm{O}$. The transported oxidant species is neutral $\mathrm{O}_{2}$. This model yields the experimentally determined pressure dependence for $k_{1}^{45}$ and shows that oxidation at the higher temperatures involves more $\mathrm{O}_{2}$ while at lower temperatures $O$ is the dominant oxidant. At the interface there is an equilibration reaction to produce $O$ from the arriving $\mathrm{O}_{2}$. A subset of this model was proposed by Blanc, ${ }^{83}$ where the initial oxidation regime, i.e., the $L_{0}, t_{0}$ regime, could be analytically accounted for by considering the reaction at the $\mathrm{Si}-\mathrm{SiO}_{2}$ interface to involve only $\mathrm{O}$. However successful this model is at explaining the initial regime, it contains neither the correct pressure dependence for $k_{1}$ nor the correct temperature dependence. On the temperature dependence, it was found that the Arrhenius plot for the $k_{1}$ displayed a concave upwards shape which is indicative of a parallel rate process. ${ }^{52}$ This is in agreement with the Ghezvan der Meulen model, but not with the Blanc model.

\section{Models based on charged species}

Several models have been proposed which incorporate the effects of charged species on the oxidation kinetics. These models are all based on the assumption that the oxidizing species possesses a negative charge. This assumption arises from results reported by Jorgensen ${ }^{4}$ claiming that the oxidation process could be enhanced or retarded by varying the direction of an electric field applied to the oxide. Recent results by Modlin and Tiller, ${ }^{14}$ discussed in Sec. II, demonstrates that Jorgensen's results are not valid, and concluded that the diffusing species is neutral for oxide thicknesses greater than $300 \AA$. Although these results seem to dispute any models based on oxidation by a charged species, they are not conclusive for oxide thicknesses below $300 \AA$. Thus it is useful to present a brief review of these models since they represent some thoughts on the source of the rapid initial regime.

The initial rapid oxidation rate observed for dry $\mathrm{O}_{2}$ ambients was recognized by Deal and Grove when they presented their model. ${ }^{37}$ To account for this behavior they applied a theory developed by Cabrera and Mott $^{12}$ to explain the initial rapid oxidation rate observed for metals. The theory predicts that space charge effects will control the oxidation until a thickness is reached, where electron transport through the oxide due to tunneling or thermionic emission is no longer possible. This thickness, given by the Debye length, is approximately $150 \AA$ for $1000^{\circ} \mathrm{C}$ dry oxidations. Deal and Grove thus concluded that the Mott-Cabrera theory provided a possible explanation for the anomolously rapid rate. However, it must be remembered that this theory was developed for the oxidation of metals where a readily available source of electrons exists at the metal surface and for a conducting oxide which could transport charge and maintain overali charge neutrality. A later model proposed by Grove $^{84}$ attributed the enhanced rate to field effects created by the formation of an $\mathrm{O}_{2}^{-}$-hole pair when the oxygen molecule enters the oxide. The greater mobility of the hole creates an additional "pull" on the ion and thus enhances the diffusion. Other models have been proposed which assume the charged oxidizing species is influenced by the presence of oxidation induced charges in the oxide ${ }^{86-89}$ Positive interfacial electronic charge, so-called fixed oxide charge $Q_{f}$ is known to exist at the $\mathrm{Si}-\mathrm{SiO}_{2}$ interface. ${ }^{85}$ One model ${ }^{87}$ proposes that this fixed oxide charge establishes an electric field within 150-200 $\AA$ of the interface region, and thus enhances the diffusion of the negatively charged oxidizing species. Incorporating an electric field assisted diffusion process into the Deal-Grove model, a linear-parabolic expression is derived with rate constants that are exponentially dependent on the charge distribution. Two fitting parameters related to the charge distribution enable a fit to the data. Models by Hamasaki $i^{88}$ and Naito et al. ${ }^{89}$ argue that the electric field established at the interface will inhibit the negatively charged oxidant species from reaching the interface. The most recent mode ${ }^{86}$ considers that the initially large number of $\mathrm{Si}$ bonds at the bare Si surface is reduced by the presence of $Q_{f}$ via the reduction in the number of holes by band bending. Neither the origin of $Q_{f}$ nor the reasons why broken bonds are crucial are treated in the model. From an assumed distribution of charge and the $1-D$ solution to Poisson's equation, a model is derived which when fitted to the oxidation data yields physically acceptable values for two parameters, the fixed oxide charge and the extent of the initially fast oxidation regime. None of these models has been tested either theoretically or experimentally against the myriad of information about $Q_{f}$ such as its orientation and ambient dependence and the commensurate effects on oxidation. Therefore, at the present such models should be treated as interesting curve fitting exercises. 


\section{Stress models}

Since the discovery of a considerable intrinsic compressive stress in oxides grown at temperatures below $950^{\circ} \mathrm{C}^{56}$ numerous models have been proposed to explain the effect of stress on the oxidation reaction. The controversy over the role of stress centers on whether stress affects the diffusion process or the reaction at the interface. Models have been proposed for both cases, but before discussing these models it is useful to present a model which describes the origin of the intrinsic stress. ${ }^{57}$ The conversion of $\mathrm{Si}$ to $\mathrm{SiO}_{2}$ involves a 2.2-fold increase in molar volume. Referring to Fig. 4 it is apparent that the oxide is constrained to the surface of the wafer, and thus the additional volume required for the formation of $\mathrm{SiO}_{2}$ must be obtained in the direction normal to the surface. At high temperatures, where viscous flow occurs, this additional volume is readily attained since the oxide can easily flow in this direction. However, at temperatures below $950^{\circ} \mathrm{C}$, where viscous flow is not observed, ${ }^{55}$ the oxide cannot attain the required molar volume, and thus an intrinsic stress develops. Whether this intrinsic stress predominantly affects the kinetic behavior of the diffusion process or the reaction to form $\mathrm{SiO}_{2}$ has been a topic of much debate. One model described below emphasizes the effect of stress on the reaction at the interface, while two other models consider the effect of stress on diffusion but reach different conclusions.

A model proposed by Irene ${ }^{31}$ introduces the effect of stress through the linear rate constant. This model contends that the lack of viscous flow at low oxidation temperatures inhibits the reaction at the interface by reducing the number of $\mathrm{Si}$ atoms that are available for reaction. The formation of $\mathrm{SiO}_{2}$ without the ability to attain the required molar volume in the normal $(z)$ direction will screen other Si atoms on the surface from reacting. A modified expression for the reaction fux is given by

$$
F_{3}=k_{r} C^{*} C_{\mathrm{Si}}^{*}
$$

where $C_{S i}^{*}$ represents the effective concentration of Si atoms avallable for reaction. This concentration is then controlled by the rate at which $\mathrm{SiO}_{2}$ can viscously relax and thereby reduce the screening effect. Treating the oxide as a Maxwell solid, the expression derived for $C_{\mathrm{Si}}^{*}$ is

$$
C_{\mathrm{si}}^{*}=k^{\prime} C_{\mathrm{Si}} \sigma_{x y} / \eta \text {, }
$$

where $\sigma_{x y}$ is the stress at the interface, $\eta$ is the viscosity of the $\mathrm{SiO}_{2}$, and $\mathrm{C}_{\mathrm{Si}}$ is the actual concentration of $\mathrm{Si}$ atoms on the

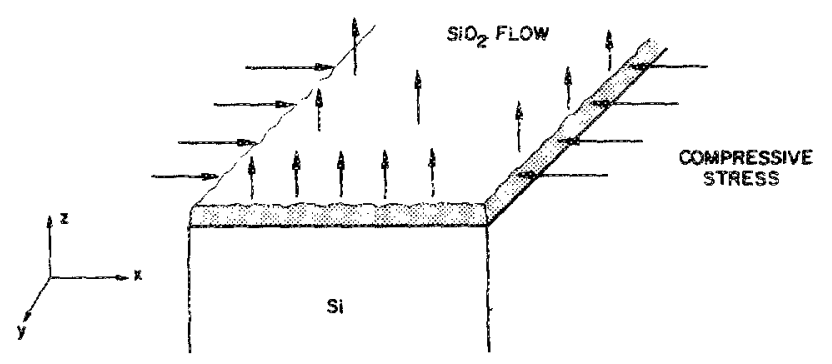

Fro. 4. The viscous flow model (from Ref. 57, Fig. 1, with permission of the Electrochemical Society). surface. Incorporating this revised fux expression into the Deal-Grove model, the integrated rate equation retains a linear-parabolic form but the linear rate constant becomes

$$
k_{\mathrm{i}}=\frac{k^{\prime \prime} C_{\mathrm{Si}} C^{*} \sigma_{x y}}{\Omega \eta}
$$

The revised linear rate constant introduces an orientation dependence through $C_{\mathrm{Si}}$ and $\sigma_{x y}$, and predicts a complex temperature behavior through $\sigma_{x y}$ and $\eta$. The behavior predicted by this model has not been confirmed because accurate values for $\sigma_{x y}$ and $\eta$ as a function of orientation and temperature have not been reported. However, the model is based on physical principles, and thus may provide an qualitative if not a quantitative description of the effects of stress on the interface reaction. This model has been used to explain the crossover in oxidation rate observed between the (110) and (111) orientations at temperatures between 800 and $1000^{\circ} \mathrm{C}$. It was suggeste ${ }^{65}$ that the initial oxidation rate is governed by a surface related property such as the number of Si-Si bonds. Beyond the crossover point, the oxide is thick enough to exert a force on the interface, arising from the intrinsic stress, which is sufficient to alter the reaction kinetics. Thus, the reaction beyond the crossover is infuenced by the stress state of the oxide.

Two models have been proposed which clairn that stress affects the diffusion process rather than the interface reaction, but the functional forms predicted by the two models for $L$ versus $t$ are quite different. The model proposed by Doremus ${ }^{58}$ asserts that the linear part of the linear-parabolic expression does not represent the reaction at the interface but instead arises from strain in the oxide. The model is based on the assumption that the only parameter controlling the oxidation rate is the diffusion of oxidant to the interface. The model assumes an exponentially decaying diffusion constant as a function of strain in the oxide:

$$
D=D_{L} \exp \left\{-k \epsilon_{0}[l-(x / L)]\right\},
$$

where $\epsilon_{0}$ represents the maximum strain located at the interface, $D_{L}$ is the diffusion constant in the outer unstrained oxide, $L$ is the total oxide thickness, and $k$ is a fitting parameter. Using this expression for the diffusion constant, a linear-parabolic equation is derived for thickness as a function of time. However, the linear term is introduced only by assuming that $\epsilon_{0}$, the maximum strain located at the $\mathrm{Si}_{-}-\mathrm{SiO}_{2}$ interface, is inversely proportional to the oxide thickness. Thus, this model only holds if this is the correct form for strain at the interface as a function of thickness. This madel does not account for the observed pressure dependence of the linear rate constant and more importantly fails to account for the fact that a linear term is required to fit the experimental data even at temperatures above $950^{\circ} \mathrm{C}$ where no strain in the oxide is observed. The other model which considers the effect of stress on the diffusion process introduces stress directly into the parabolic rate constant of the Deal-Grove model. This model, ${ }^{60}$ proposed by Fargeix and Ghibaudo, is based on a curve fitting procedure applied to plots of $d t / d L$ the inverse growth rate versus $L$. A typical plot of $d t / d L$ versus $L$ for experimental data consists of two linear regions, one extending to approximately $300 \AA$, and the other having a smaller slope extending beyond this thick- 
ness. From the Deal-Grove equation the inverse growth rate is given by

$$
\frac{d t}{d L}=\frac{2 L}{k_{p}}+\frac{l}{k_{1}}
$$

Since $k_{p}$ is proportional to $D$, the siope should be inversely proportional to $D$. Fargeix and co-workers maintain that the only way to explain the larger slope for oxide thicknesses below $300 \AA$ is by a decrease in $D$ for this region near the interface $^{59,60}$ This decreased diffusivity is attributed to stress in the oxide which is a maximum at the interface. The larger diffusion constant for thicker oxides results from a relaxation of the stress with time. At any time the diffusivity in the oxide is assumed to have the form

$$
D(x)=D_{\infty}[1-\alpha \exp (-x / \lambda)],
$$

where $\lambda$ is a damping length measured from the outer oxide surface. Using this form for the diffusivity in the DealGrove equation, they obtain a fit to the experimental data plotted as $d t / d L$ versus $L$ by correctly choosing $\alpha$ and $\lambda$ values. Although this model claims to explain the oxidation kinetics in the initial regime, there is an inconsistency in the logic they develop. The authors cite work by Marcus and Sheng ${ }^{90}$ as providing evidence for a decreased oxidation rate due to compressive stress. However, they claim this same compressive stress is responsible for a lower diffusivity near the interface and thus for the rapid initial oxidation rate observed in dry $\mathrm{O}_{2}$ oxidations. In fact the initial assumption of the model, that the difiusivity must be lower near the interface in order to explain the shape of the $d t / d L$ plot, maintains that the Deal-Grove model is valid in the initial regime when it is known that this is precisely the region where the Deal-Grove model does not apply.

It has not been clearly resolved whether stress affects the diffusion process, the reaction process or both, but it should be pointed out that if stress affects the oxidation process it is important to recognize whether the reacton is proceeding under diffusion control or reaction control before developing a model to explain the effects of stress. Clearly experimental values for stress as a function of oxidation temperature, orientation, and time are essential for evaluating these models.

\section{E. A new approach}

The most recent treatment of Si oxidation data produced a novei approach to analyzing characteristics of the oxide growth kinetics in the initial regime. ${ }^{49}$ Data collected over the temperature range $800-1000^{\circ} \mathrm{C}$ in a dry $\mathrm{O}_{2}$ ambient was analyzed in terms of the rate enhancement in the initial regime over that predicted by the Deal-Grove model. Comparing the experimentally derived $d L / d t$ versus $L$ plots with the rates predicted by the Deal-Grove model, it was determined that the "excess" growth rate could be expressed in terms of two decaying exponentials. In other words, the total growth rate is the sum of the Deal-Grove growth rate plus two additional terms which decay exponentially with thickness. One term decays rapidly with thickness from the interface and is thought to be related to surface impurities possibly introduced by wafer cleaning procedures. An intermediate phase of the enhancement is represented by a term which decays more slowly with a decay length that is roughly independent of temperature. This behavior was observed for all orientations and iemperatures investigated. Details of the characteristics of these two terms as a function of temperature, pressure, orientation, and doping level have been published. ${ }^{49}$ It was pointed out that, if these additional terms arise from additional fluxes, they must be in parallel with the fluxes enumerated in the Deal-Grove model. Possible sources for the additional fluxes are presented in the analysis. This approach to analyzing the initial regime may provide useful insight into those parameters which enhance the oxidation rate and should be incorporated into an oxidation model.

\section{SUMMARY AND FUTURE DIRECTIONS}

A variety of the most often quoted Si oxidation models have been discussed. Even many of these apparently popular ideas were eliminated from further consideration because of the lack of agreement with the presently known experimental studies of Si oxidation. Yet all of these models agree with some aspect of the oxidation phenomena and all can be made to fit the $L, t$ data. The danger of using the fit of the film growth data to the model and too few facts then becomes apparent. Essentially one arrives at necessary but not sufficient evidence. It should also be clear that the presently known facts are insufficient to determine an unambiguous and complete model for Si oxidation. It is then appropriate to end this review with a brief mention of some of the important missing parts to the Si oxidation puzzle.

On the issue of oxidant transport, it is necessary to have a measurement of the chemical diffusivity $D$ of $\mathrm{O}_{2}$ through $\mathrm{SiO}_{2}$ films with differing film growth conditions and various temperatures. At the present time only scant data exist for bulk-fused silica and none for $\mathrm{SiO}_{2}$ films. Along with $\mathrm{D}$, a measurement of the $\mathrm{O}_{2}$ solubility $S$ is required. No measurements now exist. From $D$ and $S$ the permeability $K$ can be calculated and compared with the values extracted from $L, t$ data via various models.

With regard to the chemical reaction between $\mathrm{Si}$ and $\mathrm{O}_{2}$ at the $\mathrm{Si}-\mathrm{SiO}_{2}$ interface, the kinetic order of the reaction is unknown, and the quantitative effects of the Si surface orientation and surface cleanliness are thought to be crucial parameters.

The role of intrinsic stress $\sigma_{i}$, a most promising new idea, needs further elucidation through its measurement as a function of $T, P$, Si orientation, inert annealing, $\mathrm{SiO}_{2}$ film thickness, and impurity content. Along with stress measurements the measurement of the viscous relaxation phenomenon is required so as to be able to sort among the various stress models.

The interrelationship of $Q_{f}$ and $Q_{i t}$ to stress, diffusivity, film thickness, growth and annealing conditions, and surface condition is rather vague, yet some relationships seems to exist and will undoubtedly contribute to our understanding of the $\mathrm{Si}_{-} \mathrm{SiO}_{2}$ electrical properties.

Finally, the structure of $\mathrm{SiO}_{2}$ films and the transition layer between $\mathrm{Si}$ and $\mathrm{SiO}_{2}$ will shed light on the nature of the oxidation process. 
Judging from the recent proliferation of oxidation models, it appears that the current trend in Si oxidation modeling is to speculate upon the oxidation process rather than perform the difficult measurements discussed above. Any experimental progress in determining these parameters will be of great importance in resolving the kinetic mechanism for Si oxidation, a critical concern of the semiconductor industry.

\section{ACKNOWLEDGMENT}

This work was supported in part by The Office of Naval Research.

'M. M. Atalla, E. Tannenbaum, and E. J. Scheiber, Beil Syst. Tech. J. 38, 749 (1959).

${ }^{2} 3$. R. Ligenza and W. G. Spitzer, J. Phys. Chem. Solids 14, 131 (1960).

${ }^{3}$ W. A. Pliskin and R. P. Gnall, J. Electrochem. Soc. 111, 872 (1964).

${ }^{4}$ P. J. Jorgenson, J. Chem. Phys. 37, 874 (1962).

${ }^{5} \mathrm{~K}$. N. Karube, K. Yamamoto, and M. Kamiyama, Ipn. J. Appl. Phys. 2, 11 (1963).

${ }^{6}$ E. Rosencher, A. Straboni, S. Rigo, and G. Amsel, Appl. Phys. Lett. 34, 254 (1979).

${ }^{7}$ R. Pfeffer and M. Onring, J. Appl. Phys. 52, 777 (1981).

${ }^{8}$ S. Rigo, F. Rochet, B. Agias, and A. Strabon', J. Electrochem. Soc. 129, $867(1982)$.

${ }^{9} \mathrm{~F}$. Rochet, B. Agius, and S. Rigo, J. Electrochem. Soc. 131, 914 (1984).

${ }^{10}$ J. C. Mikkelsen, Jr., A ppí. Phys. Lett. 45, 1187 (1984).

${ }^{11}$ A. G. Revesz and H. A Schaeffer, J. Electrochen. Soc. 129, 357 (1982).

${ }^{12}$ N. Cabrera and N. F. Mott, Rep. Prog. Phys. 12, 163 (1948/49).

${ }^{13}$ D. O. Raleigh, J. Electrochem. Soc. 113, 782 (1966).

${ }^{14}$ D. N. Modlin and W. A. Tiller, J. Electrochem. Soc. 132, 1659 (1985).

${ }^{15}$ B. E. Deal, J. Electrochem. Soc. 110, 527 (1963).

${ }^{16} \mathrm{H}$. Edagawa, Y. Morita, S. Maekawa, and Y. Inuishi, Jpn. \$. Appl. Phys. 2, 765 (1963)

${ }^{17}$ N. Nagasima, Jpn. J. Appl. Phys. 9, 879 (1970).

${ }^{18}$ T. W. Sigmon, W. K. Chu, E. Lugujio, and J. W. Mayer, Appl. Phys. Lett. 24, $105(1974)$.

${ }^{19}$ R. Williams and A. M. Goodman, Appl. Phys. Lett. 25, 531 (1974).

${ }^{20}$ S. I. Raider and R. Flitsch, J. Vac. Sci. Technol. 13, 58 (1976).

${ }^{21}$ T. H. DiStefano, J. Vac. Sci. Technol. 13, 856 (1976).

${ }^{22}$ J. S. Johanessen, W. E. Spicer, and Y. E. Strausser, J. Appl. Phys. 47, 3028 (1976).

${ }^{23}$ O. L. Krivanek and J. H. Mazur, Appl. Phys. Lett. 37, 392 (1980).

${ }^{24}$ M. M. Hecht, F. J. Grunthanner, P. Pianetta, L. I. Johansson, and I. Lindau, J. Vac. Sci. Technoi. A 2, 584 (1984).

${ }^{25}$ C. R. Helms, Y. E. Strausser, and W. E. Spicer, Appl. Phys. Lett. 33, 767 (1978).

${ }^{26}$ C. R. Helms, J. Vac. Sci. Technol. 16, 608 (1979).

${ }^{27} \mathrm{~J}$. M. Gibson and D. W. Dong, J. Electrochem, Soc, 127, 2722 (1980).

${ }^{28} \mathrm{~J}$. K. Srivastava and J. B. Wagner, Jr, in Proceedings of the Electrochemical Society Meeting, Cincinnati, Ohio, May 7-11, 1984.

${ }^{29}$ A. G. Revesz and R. J. Evans, J. Phys. Solids 38, 55 ! (1969).

${ }^{30}$ E. A. Irene, J. Electrochem. Soe. 125,1708 (1978).

${ }^{31}$ E. A. Irene, J. Appi. Phys. 54, $5416\{1983\}$.

${ }^{32}$ D. J. McAdam and G. W. Geil, J. Res. Natl. Bur. Stand. 28, 593 (1942).

${ }^{33}$ M. Brodsky and D. Cubicciotti, J. Am. Chem. Soc. 73, 3497 (1951)

${ }^{34}$ J. T. Law, I. Phys. Chem. 61, 1200 (1957).

${ }^{35}$ H. C. Evitts, H. W. Cooper, and S. S. Flaschen, J. Electrochem. Soc. 11R, 688 (1964).

${ }^{36}$ C. R. Fuller and F. T. Strieter, in Proceedings of the Elecrochemical Society Meeting, Toronto, Canada, May 3-7, 1964.

${ }^{37}$ B. E. Deal and A. S. Grove, J. Appl. Phys. 36, 3770 (1965).

${ }^{38}$ E. A. Irene, Semiconductor International (June, 1985), p. 97.

${ }^{39}$ Y. J. van der Meulen and N. C. Hien, J. Opt. Soc. 64, 804 (1974).

${ }^{40} \mathrm{E}$. A. Irene and Y. J. van der Meulen, J. Electrochem. Soc. 123, 1380 (1980).

${ }^{41}$ M. A. Hopper, R. A. Clarke, and L. Young, J. Electrochem. Soc. 122, $1216(1975)$.
${ }^{42}$ W. A. Pliskin, IBM J. Res. Dev. 10, 198 (1966).

${ }^{43}$ A. G. Revesz, K. H. Zaininger, and R. J. Evans, Appl. Phys. Lett. 8, 57 (1966).

${ }^{44}$ T. Nakayama and F. C. Collins, J. Electrochem. Soc. 113, 705 (1966).

${ }^{45}$ Y. J. van der Meulen, J. Electrochem. Soc. 119, 530 (1972).

${ }^{46} \mathrm{~T}$. Smith and A. J. Carlan, J. Appl. Phys. 43, 2455 (1972).

${ }^{47}$ E. A. Irene, J, Electrochem, Soc. 121, 1613 (1974).

${ }^{4 E} \mathrm{E}$. A. Irene and D. W. Dong, J. Electrochem. Soc. 125, 1146 (1978).

${ }^{49}$ H. Z. Massoud, J. D. Plummer, and E. A. Irene, J. Electrochem. Soc. 132, 1745 (1985).

${ }^{50}$ D. W. Hess and B. E. Deal, J. Electrochem. Soc. 122,579 (1975).

${ }^{51}$ R. R. Razouk, L. N. Lie, and B. E. Deal, J. Electrochem. Soc. 128, 2214 \{1981\}.

${ }^{52}$ E. A. Irene, Appì. Phys. Lett. 40, 74 (1982\%.

${ }^{53}$ J, R. Ligenza, J. Phys. Chem. 65, 201 ( (1961).

${ }^{54}$ R. Ghez and Y. J. van der Meulen, J. Electrochem. Soc. 119, $1100(1972)$.

${ }^{55}$ E. P. EerNisse, Appl. Phys. Lett. 30, 290 (1977).

56E. P. EerNisse, Appl. Phys. Lett. 35, \& (1979).

${ }^{57}$ Z. A. Irene, E. Tiemey, and J. Angillelo, J. Electrochem. Soc. 129, 2594 (1982).

${ }^{58}$ R. H. Doremus, Thin Solid Filns 122, $191(1984)$

${ }^{59}$ A. Fargeix, G. Ghibaudo, and G. Kamarinos, J. Appl. Phys. 5\&, 2878 (1983).

${ }^{60}$ A. Fargeix and G. Ghibaudo, J. Appl. Phys. 54, 7153 (1983).

${ }^{61}$ A. Fargeix and G. Ghibaudo, J. Appl. Phys. 56, 589 (1984).

${ }^{62}$ E. A. Taft, J. Electrochem. Soc. 125, 968 (1978).

${ }^{63} \mathrm{E}$. A. Krene, D. W. Dong, and R. J. Zeto, J. Electrochem. Soc. 127, 396 (1980).

${ }^{64}$ J. K. Srivistava, E. A. Irene, M. Mantini, and G. Lucousky, Appl. Phys. Lett. (submitted 1985).

${ }^{65}$ E. A. Irene, H. Z. Massoud, and E. Tiemey, J. Electrochem. Soc. (subritted 1985).

${ }^{66} \mathrm{~S}$. I. Raider and L. E. Forget, J. Electrochem. Soc. 127, 1783 (1980).

${ }^{67}$ A. G. Revesz and R. J. Evans, J. Phys. Chem. Solids 30, 551 (1969).

${ }^{67}$ D. R. Young, E. A. Irene, D. J. DiMaria, R. F. DeKeersmaecher, and H. Z. Massoud. Appl. Phys. 50, 6366 (1980).

${ }^{69} \mathrm{E}$. A. Irene and R. Ghez, J. Electrochem. Soc. 124, 1757 (1977)

${ }^{70}$ R. H. Doremus, I. Plys. Chem. 80, 1773 (1976).

${ }^{7}$ R. J. Kriegler, Y. C. Cheng, and D. R. Colton, J. Electrochem. Soc. 119, $388(1972)$.

52Y. J. van der Metulen and J. G. Cahill, J. Electron. Mater. 3, 371 (1974).

${ }^{73}$ G. J. Declerck, T. Hattori, G. A. May, J. Beaudouin, and J. D. Meindi, J. Electrochem. Soc. 322, 436 (1975),

${ }^{74}$ B. E. Deal, A. S. Grove, R. H. Srow, and C. T. Salt, J. Electrochem. Soc. 112, $308(1965)$.

${ }^{75}$ C. P. Ho and J. D. Plummer, J. Electrochem. Soc. 126, 1516 (1979).

${ }^{76}$ A. Cros, J. Phys. (Paris) 44, 707 (1983).

${ }^{77}$ F. M. d'Heurle, R. D. Frampton, E. A. Irene, H. Jiang, and C. S. Petersson, Appl. Phys. Lett. 47, 1170 (1985).

${ }^{78}$ W. A. Tiller, J. Electrochem. Soc. 128, 689 (1981).

${ }^{79} \mathrm{~B}$. Agius, M. Froment, S. Rigo, and F. Rochet, Passivity of Metals and Semiconductors, edited by M. Froment (Elsevier, New York, 1983), p. 463.

${ }^{80}$ N. F. Mott, Proc. R. Soc. London 376, 207 (1981).

${ }^{81}$ N. F. Mott, Philos. Mag. A 45, 323 (1980).

${ }^{82}$ N. F. Mott, in Proceedings of the Fifth International Symposium on Passivify, edited by M. Fromment (Elsevier, New York, 1983), p. 1.

${ }^{8: 3}$. Bianc, Appl. Phys. Lett. 33, 424 (1978).

${ }^{84}$ A. S. Grove, Physics and Technology of Semiconductor Devices (Wiley, New York, 1967), Chap. 2.

${ }^{85}$ B. E. Deal, M. \$klar, A. S. Grove, and E. H. Snow, J. Electrochem. Soc, 114, 266 (1967).

B6S. A. Schafer and S. A. Lyon, Appl. Phys. Lett. 47, 154 (1985).

${ }^{87}$ Y. Z. Lu and Y. C. Cheng, J. Appl. Phys. 56, 1608 (1984).

${ }^{88}$ M. Hamasak:, Solid State Electron. 25, 479 (1982).

${ }^{89} \mathrm{M}$. Naito, H. Homma, N. Momma, and Y. Sawahata, in Proceedings of the Electrochemical Society Meeting, Cincinnati, Ohio, May 7-11, 1984.

${ }^{50}$ R. B. Marcus and T. T. Sheng, J. Electrochem. Soc. 129, 1278 (1982).

${ }^{91}$ J. M. Aitken and E. A. Irene, Treatise on Materials Science and Technology, edited by M. Tomazawa and R. H. Doremus (Academic, New York, 1985), Vol. 26, p. 1. 